\title{
Los peces litorales del archipiélago de Juan Fernández (Chile): endemismo y relaciones ictiogeográficas
}

\author{
Germán Pequeño R. y Sylvia Sáez B. \\ Instituto de Zoología "Ernst F. Kilian", Universidad Austral de Chile \\ Casilla 567, Valdivia, Chile \\ E-mail: gpequeno@uach.cl
}

Recibido: 29 diciembre 1998; versión corregida: 4 junio 1999; aceptado: 10 septiembre 1999

\begin{abstract}
RESUMEN. A la luz del conocimiento actual de la composición ictiofaunística del archipiélago de Juan Fernández, se relacionaron aquellas especies de hábitos netamente litorales (inter y submareales), para analizar cuáles de ellas son endémicas del archipiélago y cuáles están representadas en otras regiones. Las especies endémicas alcanzan 25,5\%, aquellas representadas hacia el occidente el 19,6\%, las representadas hacia el oriente 15,6\%, las consideradas transpacíficas $9,8 \%$ y las comunes con el cercano archipiélago de Islas Desventuradas, $29,4 \%$. Se reconoce que hay un vínculo ligeramente mayor con áreas polinésicas y el Pacífico Occidental, que con las costas sudamericanas propiamente tales. Esto da lugar a pensar en un respaldo a la idea que la región zoogeográfica del Pacífico Indico-Occidental, al menos en cuanto a peces, podría propagarse hasta estas islas oceánicas.
\end{abstract}

Palabras claves: ictiogeografía, archipiélago de Juan Fernández, océano Pacífico, Chile.

\section{The littoral fishes of the Juan Fernandez archipelago (Chile): endemism and ichthyogeographical relationships}

\begin{abstract}
According to current knowledge on ichthyofaunistic composition of Juan Fernandez archipelago, those species typically littoral (inter and subtidal) were selected to analize which of them are endemic and which ones are represented in other regions. Endemic species correspond to $25.5 \%$; those represented westward are $19.6 \%$ and the eastward ones are $15.6 \%$. Transpacific species represent $9.8 \%$ of the total, whereas $29.4 \%$ of the species are in common with Desventuradas islands. A slightly higher link with Polynesian and West Pacific areas is recognised, if compared with the proper Southamerican coastlines. This supports to give background to the idea that the Indo-West Pacific zoogeographic region, at least in relation to fishes, can be extended to this oceanic islands.
\end{abstract}

Key words: ichthyogeography, Juan Fernandez archipelago, Pacific ocean, Chile.

\section{INTRODUCCIÓN}

El archipiélago de Juan Fernández se encuentra en-

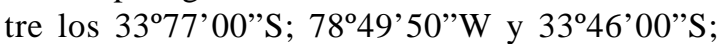
$80^{\circ} 46^{\prime} 00^{\prime \prime} \mathrm{W}$ y consta de tres emergencias: las islas Alejandro Selkirk, Robinson Crusoe y la pequeña Santa Clara, que están alrededor de $650 \mathrm{~km}$ al oeste de Valparaíso.

Los peces del archipiélago de Juan Fernández han sido estudiados desde antaño (Molina, 1782). A principio del siglo $\mathrm{XX}$, se señalaron aspectos relacionados con el grado de endemismo (Rendahl, 1921). Otro autor (Mann, 1954), ubicó a las islas en un área denominada de aguas calurosas junto con la isla de Pascua, aunque señala que esta última se encuentra influenciada por la fauna Indo-Pacífica, mientras que el archipiélago de Juan Fernández lo estaría por fauna Americano-Pacífica. Las opiniones de Mann han dejado ver su influencia prácticamente hasta el presente (Cañón y Morales, 1985). Otros autores han ubicado a las islas en un contexto biogeográfico conjunto con las islas Desventuradas (Ekman, 1967; Briggs, 1974). Recientemente, se han entregado recopilaciones sobre el total de especies 
nominales allí registradas lo cual puede implicar cambios en los conceptos ictiogeográficos (Sepúlveda y Pequeño, 1985; Sepúlveda, 1987). Otros trabajos, con nuevos registros han constituido valiosos aportes (Meléndez y Villalba, 1992). Al analizar la fauna del sector submarino al este de la isla Salas y Gómez y también la ictiofauna litoral de las islas Desventuradas, autores más recientes consideraron que la llamada fauna del Indo-Pacífico Occidental se prolongaba hasta tales enclaves, prácticamente en el sector más oriental del Pacífico Sur (Parín, 1991; Pequeño y Lamilla, 1996a).

En gran medida, las barreras señaladas por Pequeño y Lamilla (1996a) para justificar la gran diferencia ictiofaunística entre las islas Desventuradas y Chile Continental, también están operando en relación con el archipiélago de Juan Fernández, con el solo comentario que, entre esas islas y el archipiélago existen también algunas diferencias en las características físicas de las aguas superficiales, por ejemplo hacia el archipiélago de Juan Fernández son un poco más frías (Robles en Bahamonde, 1987) (Fig. 1). Del mismo modo, aquellas posibilidades de vinculación ictiofaunística con el Indo-Pacífico Occidental que operan para Islas Desventuradas, aunque algo más atenuadamente, se deben dar en el archipiélago.

Así, el problema del origen de la ictiofauna litoral de las islas del archipiélago de Juan Fernández puede encontrar una vía de respuesta, mediante el análisis de la composición taxonómica de esa ictiofauna y de sus relaciones biogeográficas.

En esta oportunidad, se presenta un análisis tomando en consideración sólo a aquellas especies de teleósteos que han sido encontradas más en relación con el sustrato litoral de las islas, que con el pélagos adyacente a ellas. Este criterio permite enfocar mejor el problema del endemismo y el de las relaciones ictiogeográficas de las islas con miras a un sentido histórico, que abra nuevas vías a la comprensión de las causas de su composición taxonómica actual.

Si se toma en cuenta que para efectos del presente estudio, el océano Pacífico Sur se puede dividir en dos sectores: a) uno muy amplio al oeste del archipiélago y b) otro mucho más estrecho (en el sentido longitudinal) hacia el este del mismo, podríamos hipotetizar que, en términos generales, el archipiélago de Juan Fernández debe tener mayor afinidad ictiofaunística con el Pacífico Occidental que con el Pacífico Oriental.
Con el fin de analizar esta posibilidad, es que se estudiará cuáles son las especies de peces netamente litorales del archipiélago y cuál es su representatividad en número y en porcentaje de aquellas especies, en diferentes partes del océano Pacífico.

\section{MATERIALES Y MÉTODOS}

Se tomó como base las listas de peces del archipiélago de Juan Fernández incluidas en trabajos recientes (Sepúlveda y Pequeño, 1985; Sepúlveda, 1987; Pequeño 1989, 1997), excluyendo aquellas especies consideradas netamente pelágicas o que no hubiesen sido capturadas en la zona litoral del archipiélago. Entonces, se efectuó una revisión de especímenes depositados en el Instituto de Zoología de la Universidad Austral de Chile, en la Universidad de Concepción, en el Museo Nacional de Historia Natural de Chile, en el Museum National d' Histoire Naturelle de París, en el Museo Británico (Londres), en la Academia de Ciencias de California (San Francisco) y en el Bernice P. Bishop Museum (Hawai). Luego, de acuerdo con otros antecedentes de la literatura (De Buen, 1961; Dyer, 1997; Hoese y Brothers, 1976; Mann, 1954; Meléndez, 1990; Meléndez y Villalba, 1992; Rendahl, 1921; Rojas y Pequeño, 1998a, 1998b; Steindachner, 1875) y de las colecciones revisadas, se procedió a establecer un cuadro de presencia ausencia por áreas de interés. Con el fin de establecer las posibles afinidades se procedió al cálculo de porcentajes. Como resultado, quedaron 51 especies.

Este criterio empleado, que permite comparaciones de ictiofauna entre ínsulas, se utilizó para saber cuántas de estas especies se encuentran en: 1) Islas Desventuradas (San Félix y San Ambrosio), 2) Isla de Pascua, 3) Isla Salas y Gómez, 4) Chile Continental, 5) Australia, 6) Nueva Zelandia, 7) Hawaii, 8) Perú y 9) Otras áreas. En esta última categoría, consideramos territorios tan variados como las islas Galápagos, Tasmania, Kermadec, Isla Norfolk, Samoa, costa de México (Pacífico) y áreas menores del Indo Pacífico.

Se consideran transpacíficas a aquellas especies que están en las riberas occidentales y orientales del océano Pacífico; las que están al occidente del archipiélago de Juan Fernández. 


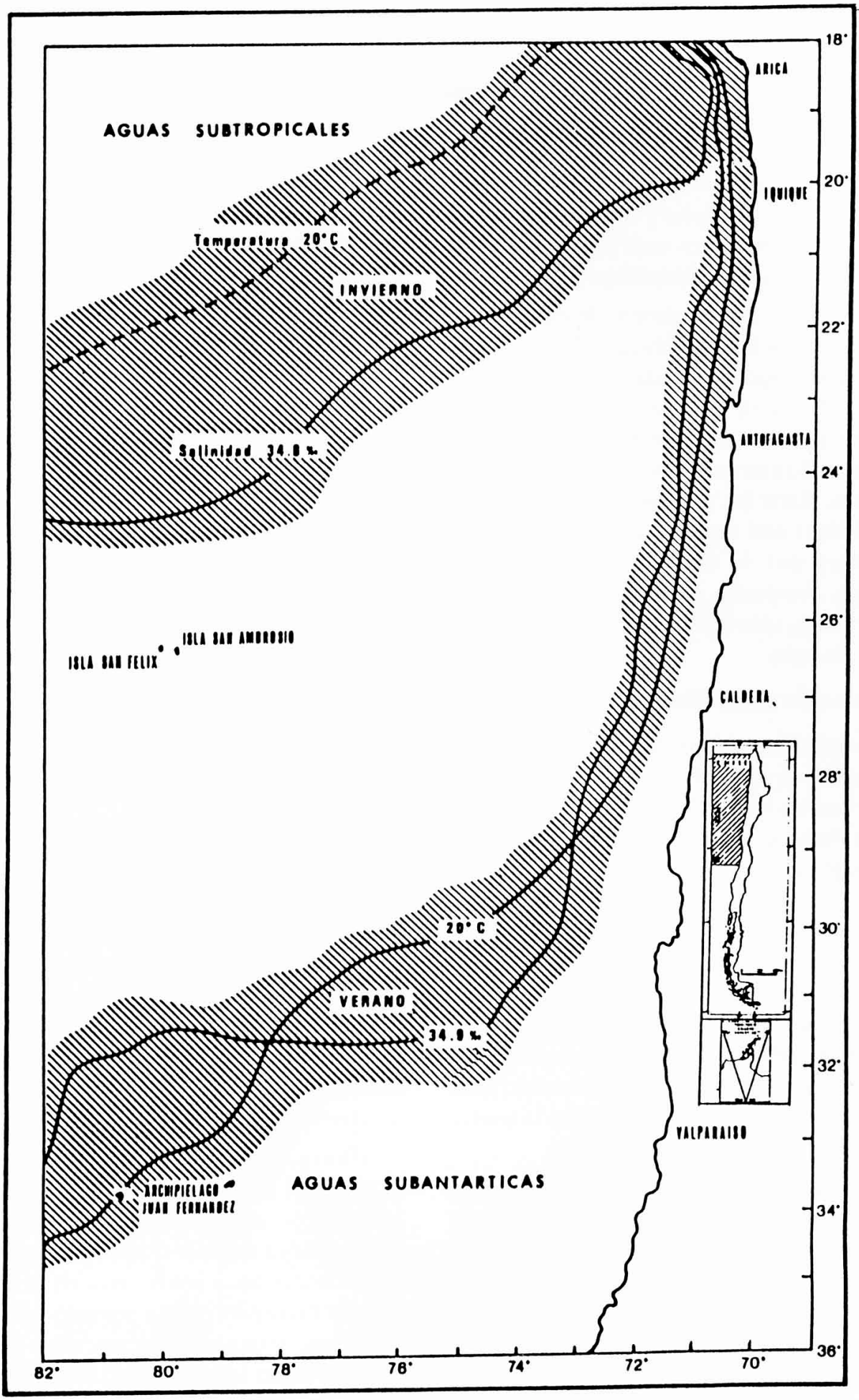

Figura 1. Ubicación del archipiélago de Juan Fernández, con indicación de isoclinas de temperatura y salinidad para invierno y verano (Tomado de Robles en Bahamonde, 1987).

Figure 1. Geographic location of Juan Fernandez archipelago, with reference of temperature and salinity isoclines during winter and summer (from Robles in Bahamonde, 1987). 


\section{RESULTADOS}

\section{Composición taxonómica}

Se ha encontrado que, entre una larga lista de especies de peces que viven en el archipiélago de Juan Fernández y sus alrededores, sólo 51 agrupadas en 27 familias, reúnen la condición de ser principalmente litorales (intermareales y submareales) y que, en el sentido biogeográfico sería la ictiofauna que mejor caracteriza a este archipiélago (Tabla 1).

La familia con mayor número de especies fue Serranidae con 6 y luego Bothidae y Scorpaenidae con 5 especies. Luego, Blenniidae con 4; Carangidae y Kyphosidae con 3; Macrorhamphosidae, Sciaenidae y Labridae con 2 y el resto, 17 (63\% entre las familias) sólo estuvo representado por una especie cada una. Entre los géneros, Paralichthys (Familia Bothidae) está representado por 5 especies, Scartichthys por 4, Girella por 3 especies, Chironemus, Scorpaena y Plectranthias con 2 especies y, el resto, sólo está representado por una especie en cada caso.

\section{Representación geográfica en otras áreas}

De las 51 especies consideradas, un porcentaje notoriamente alto se encuentra en las islas Desventuradas, que son las islas más cercanas al archipiélago de Juan Fernández, a 814 km hacia el norte (Tabla 1). Luego, se encuentra Chile Continental, seguido de la costa del Perú e isla de Pascua. Después, en orden siempre decreciente de representación, encontramos a Nueva Zelandia, isla Salas y Gómez, Australia y Hawaii. El ítem denominado otras áreas, pese a encontrarse en tercer lugar (después de Chile continental) en representatividad de especies del archipiélago de Juan Fernández, debe observarse con la precaución que implica la heterogeneidad geográfica de sus componentes.

Sin embargo, al comparar en conjunto una serie de áreas ubicadas al occidente del archipiélago de Juan Fernández (Isla de Pascua, isla Salas y Gómez, Australia y Nueva Zelandia), ocurre que de las 51 especies que hemos tenido como base en el archipiélago de Juan Fernández, 10 están presentes hacia esos lejanos enclaves mientras que 8 están representadas en las costas de Chile y Perú, reconociéndose simultáneamente que 13 son endémicas, 5 son transpacíficas, 15 comunes sólo con las islas Desventuradas y 1 es común con estas últimas y con las islas Galápagos al mismo tiempo (Tabla 2).
Aquellas especies distribuidas sólo al occidente del meridiano del archipiélago de Juan Fernández importan 19,6\%, mientras que aquellas distribuidas sólo al oriente son $15,6 \%$.

\section{DISCUSIÓN}

El presente trabajo ha intentado lograr una nueva visión en el análisis de las relaciones ictiogeográficas del archipiélago de Juan Fernández, teniendo en consideración que estas islas pueden ser lugar de respuesta a complejos problemas ictiogeográficos del Pacífico Sur (Mead, 1970; Springer, 1982). Pero, a diferencia de trabajos anteriores, este ha considerado primordialmente aquellas especies que han sido capturadas en el litoral mismo de las islas. Para asegurar la pertenencia taxonómica de las unidades consideradas, se consultaron nuevas colecciones logradas para este estudio, colecciones ya catalogadas y también se ha respetado selectas obras de ictiología, relacionadas con el tema. Con tal marco de referencia, se ha recurrido al nivel de análisis más parsimonioso: reconocer las especies del archipiélago ajustadas a lo dicho y ver cuáles de ellas se encuentran habitando otras costas y donde además, se reconocen las especies endémicas, de acuerdo con datos actuales.

Este nuevo análisis revela que el número de especies del archipiélago de Juan Fernández representadas en litorales al occidente de estas islas es ligeramente mayor que aquel representado en las costas de Chile y Perú, pese a que estas últimas están incomparablemente más cerca. Recuérdese que entre el archipiélago de Juan Fernández y Valparaíso hay $652 \mathrm{~km}$, mientras que entre ese archipiélago y las islas Desventuradas $814 \mathrm{~km}$ y el enclave más hacia occidente es la isla Salas y Gómez, a 2.697 km.

Dado que trabajos recientes (Parín, 1991; Pequeño y Lamilla 1996a, 1996b; Rojas y Pequeño, 1998a, 1998b; Rojas et al., 1998) han sugerido que la zona zoogeográfica del Indo-Pacífico Occidental se podría extender hasta zonas cercanas al archipiélago de Juan Fernández, se ha pensado que estas islas, por compartir una serie de características con aquellas otras zonas (por ejemplo, la isla Salas y Gómez e islas Desventuradas), deberían también tener un vínculo ictiogeográfico más fuerte con el Pacífico Occidental que con las costas situadas hacia su oriente. Estos planteamientos ya han sido en alguna medida respaldados por otros autores, que vinculan la 
Tabla 1. Lista sistemática de peces litorales del archipiélago de Juan Fernández.

Table 1. Systematic list of the fishes of the Juan Fernandez archipelago.

\begin{tabular}{|c|c|c|c|c|c|c|c|c|c|c|}
\hline $\begin{array}{l}\text { FAMILIA } \\
\text { ESPECIE }\end{array}$ & $\begin{array}{l}\text { Islas } \\
\text { Desven- } \\
\text { turadas }\end{array}$ & $\begin{array}{c}\text { Isla } \\
\text { de } \\
\text { Pascua }\end{array}$ & $\begin{array}{l}\text { Isla } \\
\text { Salas y } \\
\text { Gómez }\end{array}$ & $\begin{array}{l}\text { Chile } \\
\text { Conti- } \\
\text { nental }\end{array}$ & Australia & $\begin{array}{c}\text { Nueva } \\
\text { Zelandia }\end{array}$ & Hawaii & Perú & $\begin{array}{l}\text { Otras } \\
\text { áreas }\end{array}$ & Endémica \\
\hline Muraenidae & & & & & & & & & & \\
\hline $\begin{array}{c}\text { Gymnothorax porphyreus } \\
\text { (Guichenot, 1848) }\end{array}$ & + & + & & + & & & & + & + & \\
\hline Ophichthyidae & & & & & & & & & & \\
\hline $\begin{array}{c}\text { Muraenichthys chilensis } \\
\text { Mc Cosker, } 1970\end{array}$ & + & & + & & & & & & & \\
\hline Moridae & & & & & & & & & & \\
\hline $\begin{array}{l}\text { Lotella fernandeziana } \\
\text { Rendahl, } 1921\end{array}$ & + & & & & + & & & & & \\
\hline Ophidiidae & & & & & & & & & & \\
\hline $\begin{array}{c}\text { Ophidion metoecus } \\
\text { Robins, } 1991\end{array}$ & + & & & & & & & & & \\
\hline Gobiescocidae & & & & & & & & & & \\
\hline $\begin{array}{l}\text { Sicyases sanguineus } \\
\quad \text { Müller y Troschel , } 1843\end{array}$ & & & & + & & & & + & & \\
\hline $\begin{array}{l}\text { Atherinopsidae } \\
\quad \text { Odontesthes gracilis } \\
\quad \text { Steindachner, } 1898 \\
\text { Monocentridae }\end{array}$ & & & & & & & & & & + \\
\hline $\begin{array}{l}\text { Monocentridae } \\
\text { Monocentris reedi } \\
\text { Schultz, } 1956 \\
\end{array}$ & + & & + & & & & & & & \\
\hline $\begin{array}{l}\text { Trachichthyidae } \\
\text { Paratrachichthys fernandezianus } \\
\text { (Gunther, 1887) }\end{array}$ & + & & & & & & & & & \\
\hline $\begin{array}{l}\text { Macrorhamphosidae } \\
\text { Notopogon fernandezianus } \\
\text { (Delfín, 1899) }\end{array}$ & + & + & + & & & + & & & + & \\
\hline $\begin{array}{l}\text { Macrorhamphosidae } \\
\text { Macrorhamphosus scolopax } \\
\text { (Linnaeus, 1758) }\end{array}$ & + & + & + & & & + & & & & \\
\hline $\begin{array}{l}\text { Scorpaenidae } \\
\text { Cristula reticulata } \\
\text { De Buen, } 1961 \\
\end{array}$ & & & & & & & & & & + \\
\hline $\begin{array}{l}\text { Scorpaenidae } \\
\text { Helicolenus lengerichi } \\
\text { Norman, } 1937\end{array}$ & & & & + & & & & & & \\
\hline $\begin{array}{l}\text { Scorpaenidae } \\
\text { Scorpaena fernandeziana } \\
\text { Steindachner, } 1875\end{array}$ & + & & & & & & & & + & \\
\hline $\begin{array}{l}\text { Scorpaenidae } \\
\text { Scorpaena hystrio } \\
\text { Jenyns, } 1841\end{array}$ & & & & & & & + & & & \\
\hline $\begin{array}{l}\text { Scorpaenidae } \\
\text { Scorpaena uncinata } \\
\text { De Buen, } 1961 \\
\end{array}$ & + & & & & & & & & & \\
\hline $\begin{array}{l}\text { Trigilidae } \\
\text { Pterygotrigla picta } \\
\text { (Günther, 1880) }\end{array}$ & & & & & & & & & & + \\
\hline $\begin{array}{l}\text { Percichthyidae } \\
\text { Polyprion oxygeneios } \\
\text { (Schneider, 1801) } \\
\end{array}$ & + & + & + & + & & + & & & + & \\
\hline $\begin{array}{l}\text { Serranidae } \\
\quad \text { Callanthias platei } \\
\quad \text { Steindachner, } 1898\end{array}$ & + & & & & & & & & + & \\
\hline
\end{tabular}




\begin{tabular}{|c|c|c|c|c|c|c|c|c|c|c|}
\hline $\begin{array}{l}\text { FAMILIA } \\
\text { ESPECIE }\end{array}$ & $\begin{array}{l}\text { Islas } \\
\text { Desven- } \\
\text { turadas }\end{array}$ & $\begin{array}{c}\text { Isla } \\
\text { de } \\
\text { Pascua }\end{array}$ & $\begin{array}{c}\text { Isla } \\
\text { Salas y } \\
\text { Gómez }\end{array}$ & $\begin{array}{l}\text { Chile } \\
\text { Conti- } \\
\text { nental }\end{array}$ & Australia & $\begin{array}{l}\text { Nueva } \\
\text { Zelandia }\end{array}$ & Hawai & Pert & $\begin{array}{l}\text { Otras } \\
\text { áreas }\end{array}$ & Endémica \\
\hline
\end{tabular}

Serranidae

Caprodon longimanus

(Günther, 1859)

\section{Serranidae}

Hypoplectrodes semicinctum

(Valenciennes, 1833)

Serranidae

Paralabrax humeralis

(Valenciennes, 1828)

Serranidae

Plectranthias exsul

Heemstra y Anderson, 1983

\section{Serranidae}

Plectranthias lamillai

Rojas y Pequeño, 1998

Carangidae

Pseudocaranx chilensis

(Guichenot, 1848)

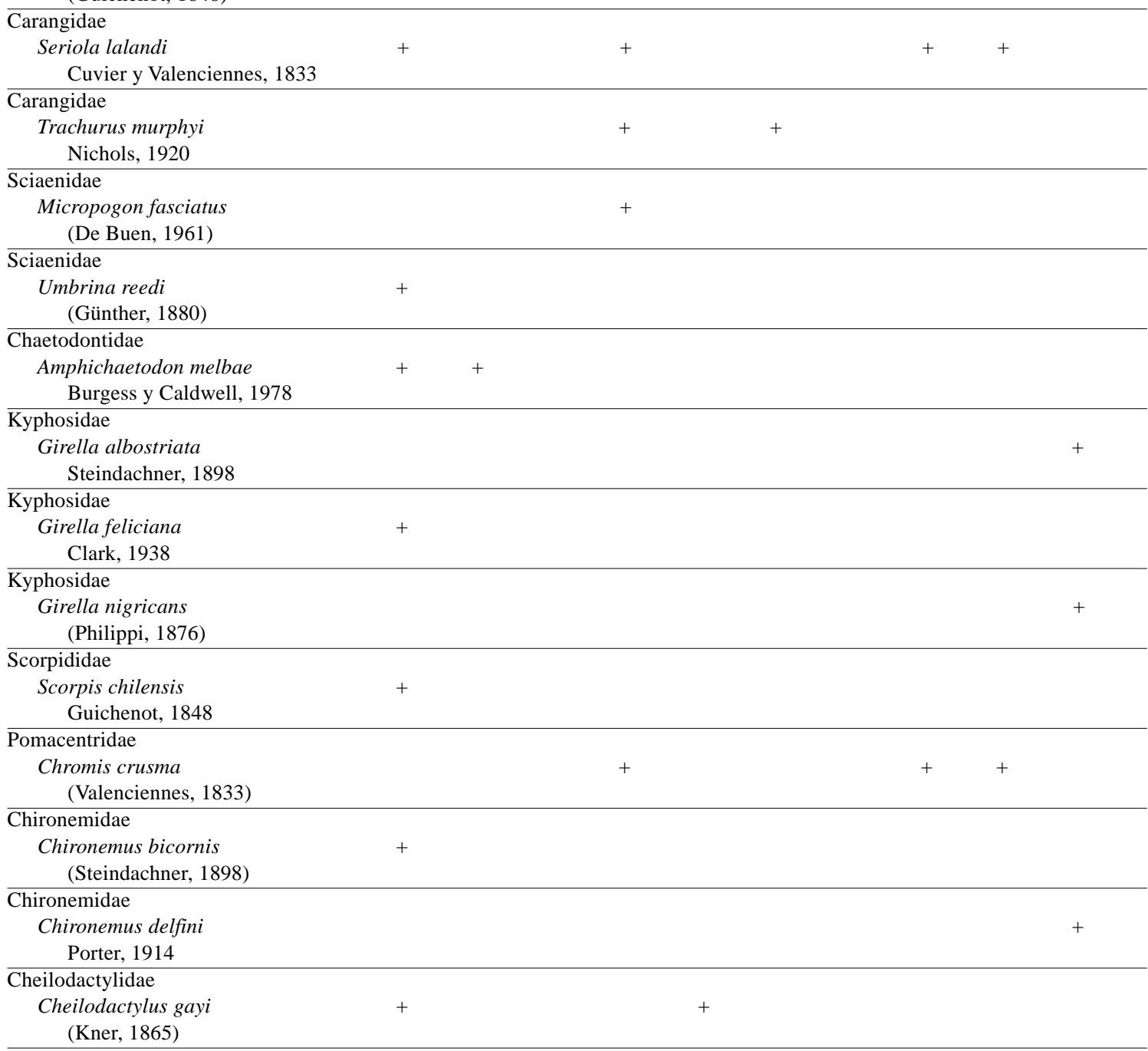




\begin{tabular}{|c|c|c|c|c|c|c|c|c|c|}
\hline $\begin{array}{l}\text { FAMILIA } \\
\text { ESPECIE }\end{array}$ & $\begin{array}{l}\text { Islas } \\
\text { Desven- } \\
\text { turadas }\end{array}$ & $\begin{array}{c}\text { Isla } \\
\text { de } \\
\text { Pascua }\end{array}$ & $\begin{array}{c}\text { Isla } \\
\text { Salas y } \\
\text { Gómez }\end{array}$ & $\begin{array}{l}\text { Chile } \\
\text { Conti- } \\
\text { nental }\end{array}$ & Australia & $\begin{array}{c}\text { Nueva } \\
\text { Zelandia }\end{array}$ & Hawai & Per & $\begin{array}{l}\text { Otras Endémica } \\
\text { áreas }\end{array}$ \\
\hline
\end{tabular}

Latrididae

Mendosoma fernandeziana

Guichenot, 1848

Labridae

Pseudolabrus gayi

(Valenciennes, 1839)

Labridae

Malapterus reticulatus

Valenciennes, 1839

Pinguipedidae

Parapercis dockinsi

(Mc Cosker, 1971)

Blenniidae

Scartichthys fernandezensis (Clark, 1938)

Blenniidae

Scartichthys rubropunctatus

(Valenciennes, 1836)

Blenniidae

Scartichthys variolatus

(Valenciennes, 1836)

\section{Blenniidae}

Scartichthys viridis

(Valenciennes, 1836)

Gobiidae

Paratrimma nigrimenta Hoese y Brothers, 1976

Bothidae

Paralichthys adspersus

(Steindachner, 1867)

Bothidae

Paralichthys coeruleosticto

Steindachner, 1898

Bothidae

Paralichthys fernandezianus

Steindachner, 1905

Bothidae

Paralichthys hilgendorfi

Steindachner, 1905

Bothidae

Paralichthys schmitti

Ginsburg, 1933 
Tabla 2. Peces litorales de Juan Fernández, según su distribución geográfica al occidente, al oriente, transpacíficas, endémicas y de sola compartición con las islas Desventuradas.

Table 2. The littoral fishes of Juan Fernandez, according to their geographic distribution to the west, the east, transpacifics, endemics and shared with Desventuradas islands only.

\begin{tabular}{|ll|}
\hline a) Al occidente & b) Al oriente \\
\hline Muraenicthtys chilensis & Sicyases sanguineus \\
Lotella fernandeziana & Helicolenus lengerichi \\
Monocentris reedi & Paralabrax humeralis \\
Notopogon fernandezianus & Seriola lalandi \\
Macrorhamphosus scolopax & Micropogon fasciatus \\
Scorpaena hystrio & Scartichthys rubropunctatus \\
Callanthias platei & Scartichthys viridis \\
Hypoplectrodes semicinctum & Paralichthys adspersus \\
Plectranthias exsul & \\
Cheilodactylus gayi & \\
\hline & \\
$\mathrm{n}=10$ especies (19,6\%) & $\mathrm{n}=8$ especies (15,6\%) \\
\hline
\end{tabular}

\begin{tabular}{|c|c|c|}
\hline c) Sólo transpacífica & d) Endémicas & $\begin{array}{l}\text { e) Compartidas sólo con las } \\
\text { islas Desventuradas }\end{array}$ \\
\hline Gymnothorax porphyreus & Odontesthes gracilis & Ophidion metoecus \\
\hline Polyprion oxigeneios fernandezianus & Cristula reticulata & Paratrachichthys fernandezianus \\
\hline Caprodon longimanus & Pterygotrigla picta & Scorpaena fernandeziana $(*)$ \\
\hline Trachurus murphyi & Plectranthias lamillai & Scorpaena uncinata \\
\hline \multirow[t]{11}{*}{ Chromis crusma } & Girella albostriata & Umbrina reedi \\
\hline & Girella nigricans & Girella feliciana \\
\hline & Pseudocaranx chilensis & Scorpis chilensis \\
\hline & Chironemus delfini & Chironemus bicornis \\
\hline & Mendosoma fernandiazana & Pseudolabrus gayi \\
\hline & Paralichthys coeruleosticta & Malapterus reticulatus \\
\hline & Paralichthys fernandezianus & Parapercis dockinsi \\
\hline & Paralichthys hilgendorfi & Scartichthys fernandezensis \\
\hline & Paratrimma nigrimenta & Scartichthys variolatus \\
\hline & & Paralichthys schmitti \\
\hline & & Amphichaetodon melbae \\
\hline $\mathrm{n}=5$ especies $(9,8 \%)$ & $\mathrm{n}=13(25,5 \%)$ & especies $(29,4 \%)$ \\
\hline
\end{tabular}

(*) También en islas Galápagos 
distribución de especies del oeste hacia al este, a problemas abióticos de las aguas y a fenómenos geológicos de emergencia, submersión, erosión y otros (Lavenberg, 1992). El alto porcentaje de especies compartido sólo con islas Desventuradas, se debe observar a la luz del fuerte vínculo que los géneros involucrados tienen con el Pacífico Occidental. Por otro lado, se reconoce que algunas especies atribuidas en común con Chile-Perú (por ejemplo: S. sanguineus; $S$. viridis), requieren estudios taxonómicos más detallados, que podría cambiar algunas cifras.

El estudio ha coincidido con esta última hipótesis y las causas pueden ser similares a las dadas en el caso de las islas Desventuradas: las especies netamente litorales del archipiélago de Juan Fernández (excepto las transpacíficas) requieren de un sustrato cercano para su sobrevivencia, además de temperaturas relativamente más altas, que aquellas de la corriente de Humboldt, que se interpone entre Juan Fernández y el continente. Se puede agregar también, que las especies netamente litorales como las analizadas se caracterizan porque sus habilidades natatorias son, en general, menores que aquellas de las especies pelágicas, lo cual es un impedimento para recorrer distancias largas como las existentes entre las islas y los continentes. De allí que la posibilidad de transporte de huevos y juveniles por corrientes, algas a la deriva o mecanismos similares, cobra un gran interés. Las corrientes superficiales son en gran medida responsables por la gran diferencia entre la extensión y la riqueza de la fauna marina tropical a ambos lados del Pacífico (Hubbs y Rosenblatt, 1961).

Aún cuando existen opiniones sobre la posible edad de las islas del archipiélago de Juan Fernández, aproximadamente 2,5 a 6,0 millones de años (Newman y Foster, 1983; Stuessy et al. 1984), resulta muy complejo señalar las posibles causas de la presencia en ellas de un grupo tan diverso de especies. Algunas que deben poseer larvas leptocéfalas (aún desconocidas), pueden haber sido transportadas a grandes distancias desde el Pacífico Occidental, como podría ser el caso de Muraenichthys y Gymnothorax. Otros pueden haber viajado con algas u otros objetos flotantes a la deriva y otras, incluso pueden haber sido transportadas involuntariamente por el hombre, como se ha sugerido para un posible transporte este a oeste del "pejesapo" Sicyases sanguineus, desde Chile central (Pequeño, 1989).
De acuerdo con los antecedentes actuales, el grado de endemismo parece ser menor al 50\% indicado en forma aproximada por Rendahl (1921) y mencionada por Ekman (1967), pues hemos encontrado alrededor de $25,5 \%$. Una causa de variación en las cifras sobre endemismo puede estar motivada en hallazgos de algunas especies en otras áreas en los últimos años, como por ejemplo en las islas Desventuradas que, pese a tener un alto porcentaje en común, aún mantiene diferencias todavía no bien estudiadas y sobre las cuales los factores abióticos pueden ser causa mayor. También es necesario recordar que los estudios taxonómicos y sistemáticos pueden influir cuando se encuentran nuevas sinonimias o, mediante el caso opuesto, que implican descubrir más de una especie donde se creyó que sólo había una. Aunque Ekman (1967) señala que las islas "deben aparentemente ser incluidas en la región templado-cálida, como un apéndice claramente independiente" y que "el elemento sudamericano es también considerable, siendo sin embargo el elemento sudamericano específico no más rico en especies que el circumtemplado más general". Pero, su falta de cuantificación dejó un vacío que es requerido para dar mayor consistencia a sus aseveraciones. Esto no sólo porque la evidencia es entonces reconocida, sino porque además la composición taxonómica, permite un análisis más acabado a nivel de especies.

Pese a ser isla de Pascua y el archipiélago de Juan Fernández mejor conocidos ictiogeográficamente que islas Desventuradas e isla Salas y Gómez, se piensa que nuevas exploraciones pueden proporcionar también nuevos datos, probablemente incrementando el número de especies o ayudando a resolver algunas dudas, como por ejemplo las planteadas por géneros con números de especies relativamente altos, conviviendo en un perímetro algo limitado, como ocurre con Scartichthys y Paralicthys o bien en relación con nuevos cambios sistemáticos o taxonómicos.

Para lograr una comprensión más completa y profunda de los orígenes geográficos de la ictiofauna del archipiélago de Juan Fernández, se cree que será necesario entrar en el análisis biosistemático y biogeográfico de cada uno de los taxa que componen tan interesante conjunto. 


\section{AGRADECIMIENTOS}

Al Profesor Nibaldo Bahamonde N. (Academia de Ciencias, Chile), por su constante aliento para estas investigaciones; al Dr. Walter Fischer (FAO, Roma) por su apoyo desinteresado; al Dr. Jean-Claude Hureau (Museum National d' Historie Naturelle, París) por las facilidades para revisar colecciones de peces; al Dr. Mark W. Westneat (Field Museum of Natural History, Chicago), por su invitación a participar en una expedición al archipiélago de Juan Fernández. Estos son resultados parciales del Proyecto S-96-04 de la Universidad Austral de Chile y del Grant 5257-96 de la National Geographic Society.

\section{REFERENCIAS}

Albert, F. 1898. Fauna Chilena. La langosta de Juan Fernández y la posibilidad de su propagación en la costa chilena. Rev. Chil. Hist. Nat., 2: 5-11.

Bahamonde, N. 1987. San Félix y San Ambrosio, las islas llamadas Desventuradas pp. 85-100. En: J.C. Castilla (ed.). Islas Oceánicas Chilenas: Conocimiento científico y necesidades de investigaciones. Edic. Univ. Católica de Chile, Santiago.

Briggs, J.C. 1974. Marine zoogeography. Mc GrawHill, New York, 475 pp.

Cañón, J.R y E. Morales. 1985. Geografía de Chile. Geografía del Mar Chileno Instituto Geográfico Militar, 9: 1-244.

De Buen, F. 1961. Peces Chilenos. Familias Alepocephalidae, Muraenidae, Sciaenidae, Scorpaenidae, Liparidae y Bothidae. Montemar (Continuación de la Rev. Biol. Mar.), 1: 1-52.

Dyer, B.S. 1997. Phylogenetic revison of Atherinopsinae (Teleostei; Atherinopsidae), with comments on the systematics of the South American freshwater fish genus Basilichthys Girard. Misc. Publ. Mus. Zool., Univ. Michigan, 185: 1-64.

Dyer, B.S. 1998. Phylogenetic systematics and historical biogeography of the neotropical silverside Family Atherinopsidae (Teleostei; Atheriniformes). pp. 519-536. En: L.R. Malabarba, R.E. Reis, R.P. Vari, Z.M. Lucena y C.A.S. Lucena (eds.). Phylogeny and classification of neotropical fishes. Porto Alegre, Edipuers, 603 pp.
Ekman, S. 1967. Zoogeography of the sea. Sidgwick and Jackson, London, 417 pp.

Hoese, D.F. y E.B. Brothers. 1976. Paratrimma, a new genus of gobid fishes and two new species. Copeia, 1976(3): 494-497.

Hubbs, C.L. y R.H. Rosenblatt. 1961. Effects of the equatorial currents of the Pacific on the distribution of fishes and other marine animals. pp 340-341 En: Abstracts of symposium papers. Tenth Pacific Science Congress. Pacific Sci. Ass. (ed.), Honolulu, Hawaii.

Lavenberg, R.J. 1992. A new moray eel (Muraenidae: Gymnothorax) from oceanic island of the South Pacific. Pacific Sci., 46(1): 58-67.

Mann, G. 1954. La vida de los peces en aguas chilenas. Ministerio de Agricultura, Instituto de Investigaciones Veterinarias y Univ. de Chile. Santiago de Chile, 343 pp.

Mead, G.W. 1970. A history of South Pacific fishes. pp. 236-251. En: Scientific exploration of the South Pacific. Nat. Acad. Sci., Washington, D.C.

Meléndez, C.R. 1990. Chironemid fishes from Juan Fernández archipelago and Desventuradas islands, Chile. (Perciformes: Chironemidae). Rev. Biol. Mar., Valparaíso, 25(2): 83-92.

Meléndez, C.R. y C. Villalba. 1992. Nuevos registros y antecedentes para la ictiofauna del archipiélago de Juan Fernández, Chile. Estud. Oceanol., 11: 3-29.

Molina, J.I. 1782. Saggio sulla storia naturale del Cile. Bologna, $367 \mathrm{pp}$.

Newman, A. y B.A. Foster. 1983. The Rapanuian faunal district (Easter and Sala y Gómez). En: In search of ancient archipelagos. Bull. Mar. Sci., 33: 633-644.

Parín, N.V. 1991. Fish fauna of the Nazca and Sala y Gómez submarine ridges, the esternmost outpost of the Indo-West Pacific zoogeographic region. Bull. Mar. Sci., 49(3): 671-683.

Pequeño, G. 1989. Peces de Chile. Lista sistemática revisada y comentada. Rev. Biol. Mar., Valparaíso, 24(2): 1-132.

Pequeño, G. 1997. Peces de Chile. Lista sistemática revisada y comentada: Addendum. Rev. Biol. Mar. Oceanogr, Valparaíso, 32(2): 77-94. 
Pequeño, G. y J. Lamilla. 1996a. Desventuradas islands, Chile: the easternmost outpost of the IndoWest Pacific zoogeographic region. Rev. Biol. Trop., 44(2): 887-889.

Pequeño, G. y J. Lamilla. 1996b. Peces de la Familia Serranidae en las Islas Desventuradas, Chile (Osteichthyes, Perciformes). Bol. Soc. Biol. Concepción, Chile, 67: 23-32.

Rendahl, H. 1921. The fishes of the Juan Fernández islands. En: C. Skottsberg (ed.). The Natural History of Juan Fernández and Easter Island. Uppsala, 3: 49-58.

Rojas, R. y G. Pequeño. 1998a. Peces Serránidos de la isla Alejandro Selkirk, archipiélago de Juan Fernández, Chile (Pisces: Serranidae): Análisis ictiogeográfico. Invest. Mar., Valparaíso, 26: 41-58.

Rojas, R. y G. Pequeño. 1998b. Plectranthias lamillai, a new Anthiine fish species (Perciformes, Serranidae) from the Juan Fernández archipelago, Chile. Sci. Mar., 62(3): 203-209.

Rojas, R., S. Palma y G. Pequeño. Food of the grouper Caprodon longimanus (Gunther, 1859) from the Alejandro Selkirk Island, Chile (Perciformes: Serranidae). Rev. Biol. Trop., 46(4): 937-942.
Sepúlveda, J.I. 1987. Peces de las islas oceánicas chilenas. pp. 225-245. En: J.C. Castilla (ed). Islas oceánicas chilenas: Conocimiento científico y necesidades de investigación. Edic. Univ. Católica de Chile, Santiago.

Sepúlveda, V. y G. Pequeño. 1985. Fauna íctica del archipiélago de Juan Fernández. pp. 81-91. En: P. Arana (ed). Investigaciones Marinas en el Archipiélago de Juan Fernández. Esc. Ciencias del Mar, UCV, Valparaíso.

Springer, V.G. 1982. Pacific plate biogeography, with special reference to shorefishes. Smith. Contr. Zool., 367: 4-182.

Steindachner, F. 1875. Die Fische von Juan Fernandez in dem Sammlungen des Wiener Museums Über einige Fischarten von der ost und West-Küste SüdAmerikas. Ichth. Beiträge 2. Sitzb. Akad. Wiss. Wien., 71.

Stuessy, T.F., K.A. Foland, J.F. Sutter, R.W. Sanders y M. Silva. 1984. Botanical and geological significance of potassium-argon dates from the Juan Fernandez islands. Science, 225: 49-51. 
